etrueream

* Gated RapidArc

* Five Photon Beam

* High Dose Rate : 2400MU/min

* Supervisor System

* 4D CBCT and Cine Mode

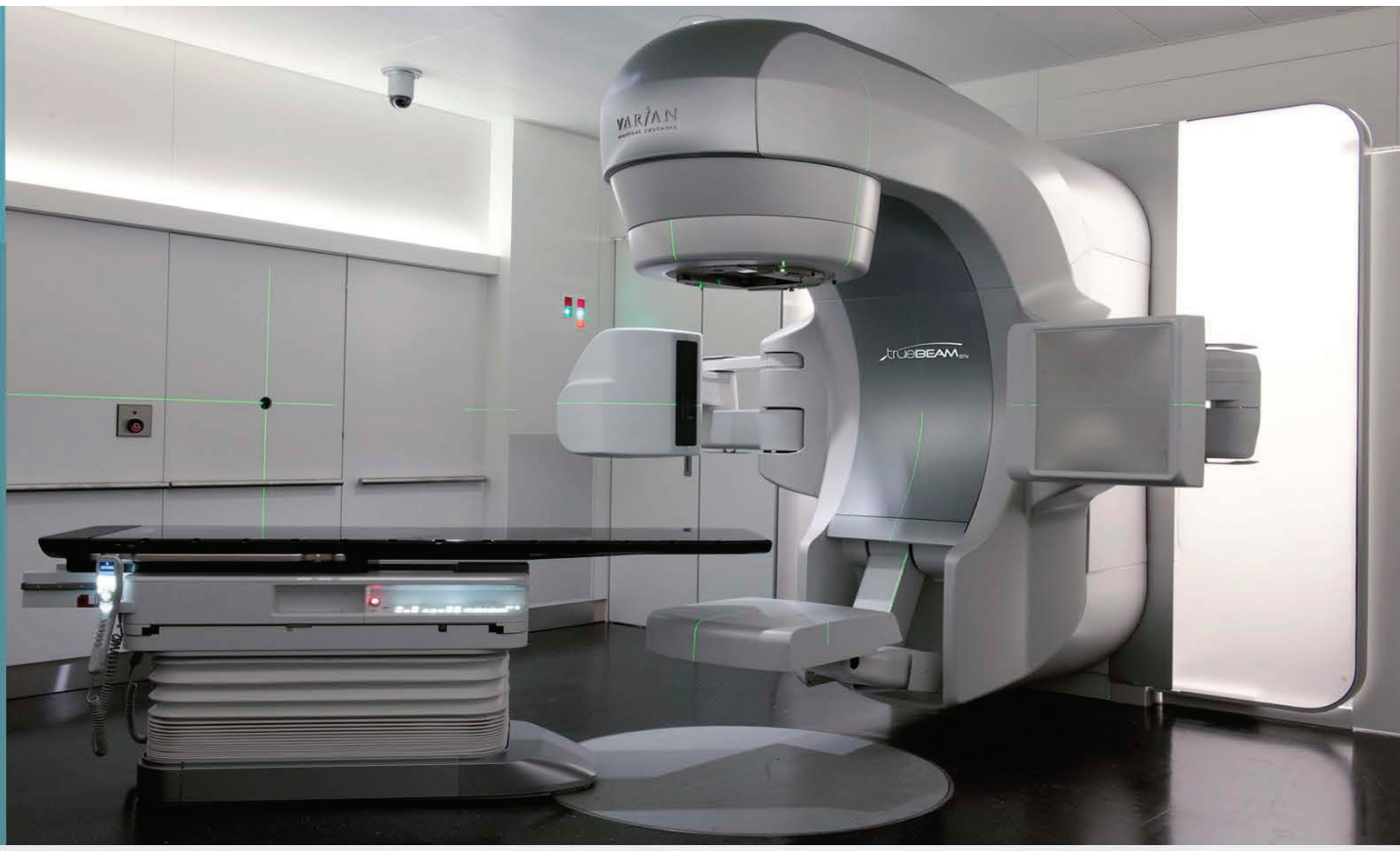

\section{-Vit:ol $\widehat{B E A M}$}

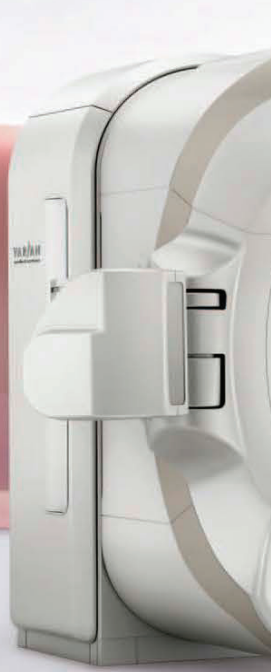

\section{VITAL TOGETHER}

Your oncology expertise combined with the innovative power of VitalBeam can help take your clinic to the next level now and into the future.
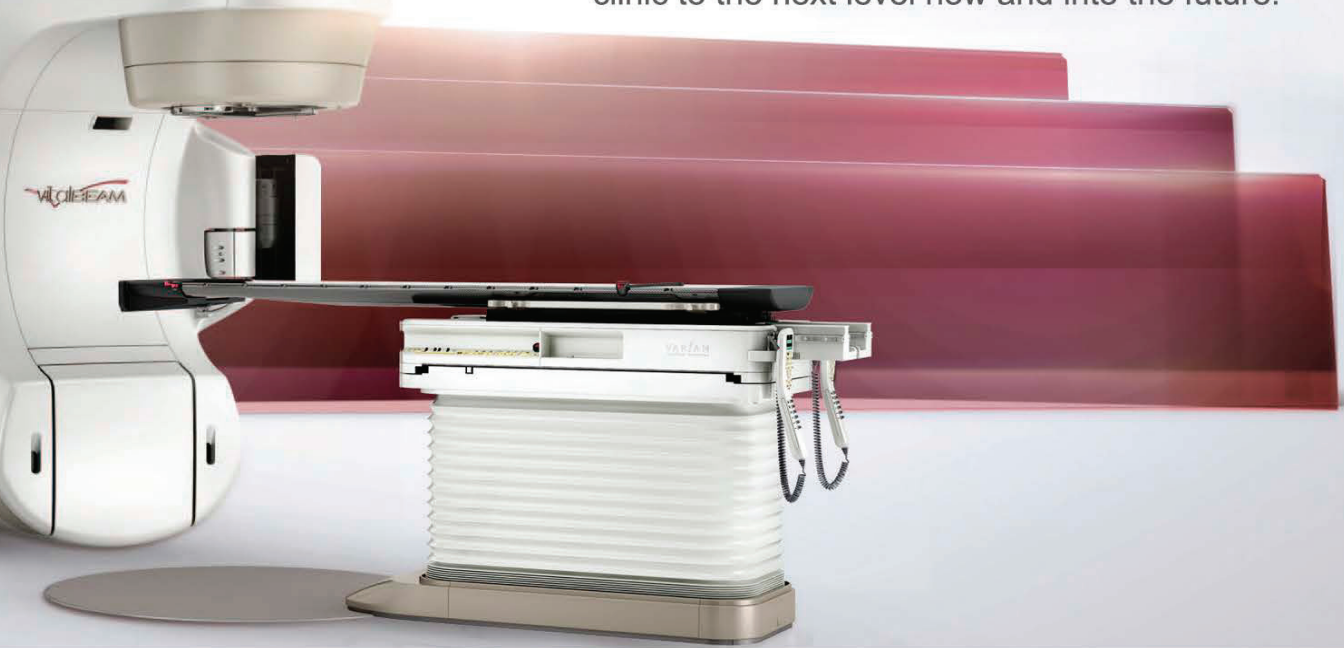

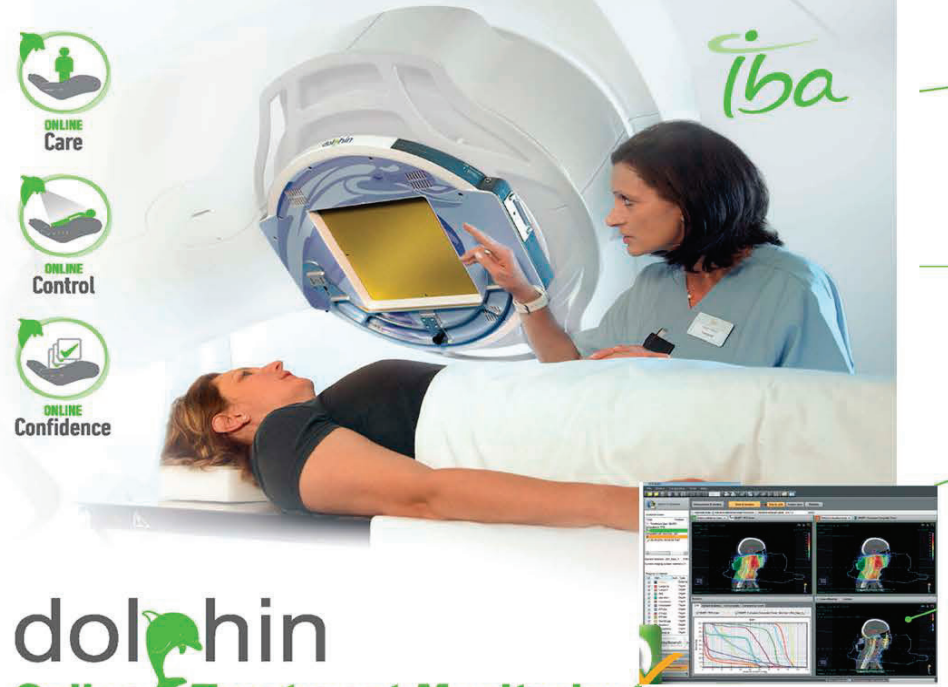

Online Treatment Monitoring

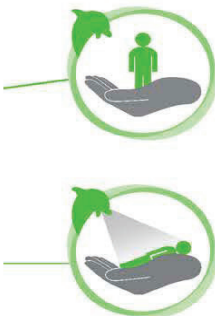

Offer your patients more care and comfort

to give them hope, belief and "the will to fight."

Be certain you have control over your

patients' safety against accidents!

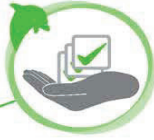

Have the confidence and peace of mind

knowing that your treatment is accurate

until the last fraction!
$2 \underset{\text { Larparation }}{2}$

서울시 종로구 인사동 194-27

TEL : 02) 2003-8441

FAX : 02) 737-4677 


\title{
Initial Experience of Patient-Specific QA for Wobbling and Line-Scanning Proton Therapy at Samsung Medical Center
}

\author{
Kwanghyun Jo, Sung Hwan Ahn, Kwangzoo Chung, Sungkoo Cho, Eun Hyuk Shin, Seyjoon Park, \\ Chae-Seon Hong, Dae-Hyun Kim, Boram Lee, Woojin Lee, Doo Ho Choi, Do Hoon Lim, Hong Ryull Pyo, \\ Youngyih Han \\ Department of Radiation Oncology, Samsung Medical Center, Sungkyunkwan University School of Medicine, Seoul, Korea
}

Received 14 December 2018 Revised 12 March 2019

Accepted 12 March 2019

Corresponding author Youngyih Han

(youngyih@skku.edu)

Tel: 82-2-3410-2604

Fax: 82-2-3410-2619
Purpose: To report the initial experience of patient-specific quality assurance ( $p Q A)$ for the wobbling and line-scanning proton therapy at Samsung Medical Center.

Materials and Methods: The pQA results of 89 wobbling treatments with 227 fields and 44 linescanning treatments with 118 fields were analyzed from December 2015 to June 2016. For the wobbling method, proton range and spread-out Bragg peak (SOBP) width were verified. For the line-scanning method, output and two-dimensional dose distribution at multiple depths were verified by gamma analysis with $3 \% / 3 \mathrm{~mm}$ criterion.

Results: The average range difference was $-0.44 \mathrm{~mm}$ with a standard deviation (SD) of $1.64 \mathrm{~mm}$ and $0.1 \mathrm{~mm}$ with an SD of $0.53 \mathrm{~mm}$ for the small and middle wobbling radii, respectively. For the line-scanning method, the output difference was within $\pm 3 \%$. The gamma passing rates were over 95\% with $3 \% / 3 \mathrm{~mm}$ criterion for all depths.

Conclusions: For the wobbling method, proton range and SOBP width were within the tolerance levels. For the line-scanning method, the output and two-dimensional dose distribution showed excellent agreement with the treatment plans.

Keywords: Proton therapy, Patient specific QA, Wobbling, Line-scanning

\section{Introduction}

Since the introduction of proton beam therapy for medical use, the number of proton therapy facilities has been increasing worldwide. According to PTCOG (Particle Therapy Co-Operative Group) patient statistics, ${ }^{1)}$ over 170,000 patients have been treated by proton therapy from 1954 to 2017 and 79 facilities are in operation. Main reason for the increasing number of proton facilities is the dosimetric benefit of proton therapy which does not have an exit dose due to the characteristics of Bragg peaks. It opens a new way to save normal tissue by the reducing radiation dose to an area close to the target which would lower the risk of necrosis, mucositis, radiation pneumonitis, and secondary malignancy. ${ }^{2,3)}$

It, however, should be aware of that proton therapy is very sensitive to uncertainty because of the sharp dose gradient at the distal region of the proton beam. The systematic uncertainties relevant to proton range include the inaccuracy of algorithm in dose computation, the uncertainty in the determination of stopping power from the computed tomography (CT) data, and daily machine status. ${ }^{4)}$ To verify 
whether the uncertainty is within the tolerance, patientspecific quality assurance (pQA) is necessary. It is a procedure delivering a dose with machine parameters identical to the patient treatment plan to ensure the consistency of a system and the accuracy of the delivered dose. The range, output, and two-dimensional dose distribution are verified by comparing the measurements with the treatment plan.

This work presents statistics and characteristics of the wobbling and the line-scanning pQA. Many institutions reported the result of $\mathrm{pQA}$ for the spot-scanning ${ }^{5-9)}$ but the pQA result of the wobbling and the line-scanning have not been reported yet. Even a majority of the proton treatments are shifted to the spot-scanning, there are users to have the wobbling and the line-scanning and this could help them by sharing the initial experience of pQA.

\section{Materials and Methods}

\section{Proton therapy system at Samsung Medical Center}

Proton therapy system (Sumitomo Heavy Industry, SHI, Japan) at Samsung Medical Center consisted of one cyclotron and two rotating gantries ${ }^{10)}$; one was equipped with a multipurpose nozzle for both capable of the wobbling and the line-scanning treatments and the other was a scanning dedicated nozzle only for the line-scanning treatment. The wobbling method ${ }^{11,12)}$ was analogous to the double scatter- ing method of the IBA system. The initial pencil beam was rotated laterally along an elliptical track 11 times a second and let to pass a scatterer to form a blurred Gaussian beam, an example was given in Fig. 1. The major and minor radius of the ellipse, hereafter referred to as a wobbling radius (WR), varied depending on energy and field size. The wobbling method had three categories of field size to optimize the wobbling radius: a "small" (diameter $<11 \mathrm{~cm}$ ), a "middle" (11 cm $\leq$ diameter $<16 \mathrm{~cm})$, and a "large" (diameter $<16$ $\mathrm{cm})$. We did not use a large WR beam because more fineretuning was necessary to meet the accuracy required for clinical applications. Wobbling delivery method produced a spread-out Bragg peak (SOBP) by a ridge filters which was specifically designed to spread the proton energy from low to high with appropriate weights. The range of the proton beam was defined as the distal $90 \%$ of dose level and the SOBP width was defined as the length between points at distal $90 \%$ and at proximal $95 \%$ of dose level, which was normalized at mid SOBP.

The line-scanning method was different from spotscanning in the following sense. It used the line-segment, a continuous proton beam irradiation along a line, instead of using a proton spot beam. The intensity modulation was achieved by controlling both the dose rate and scanning speed, while for spot-scanning it was done by changing MU for each beam position. It was found that both methods lead to nearly identical results. ${ }^{13)}$

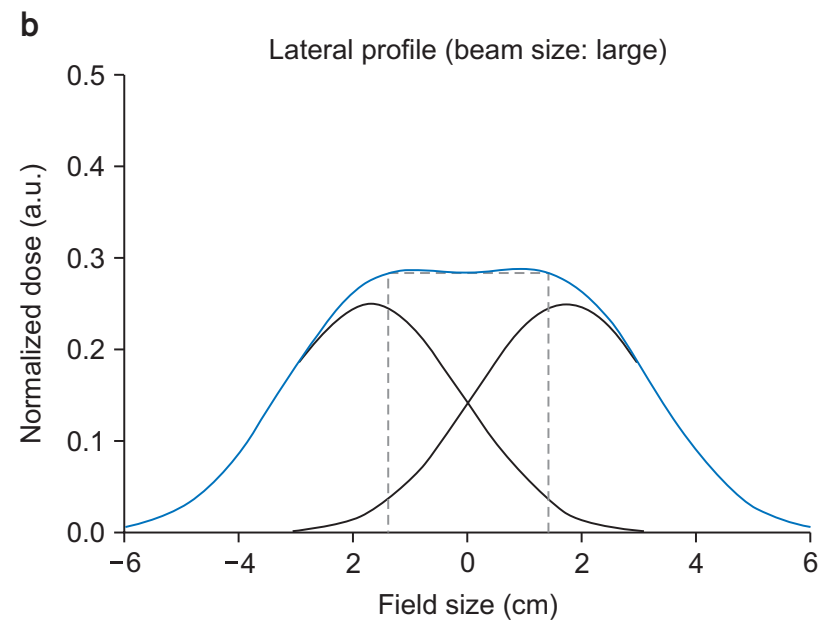

Fig. 1. One dimensional profile of wobbling beam is presented as an example. (a) The Gaussian beam size is small, the wobbling radius is small. (b) The Gaussian beam size is large, wobbling radius is large. The amplitude is normalized by the initial amplitude of Gaussian beam. The wobbling radius is fine-tuned in order to make flat central region. 
The oncology information system (OIS) is Mosaiq (Elekta, Stockholm, Sweden), and the treatment planning system (TPS) is Raystation (Raysearch Lab. AB., Sweden). Both software applications can be accessed remotely by using Citrix (Citrix system, US).

\section{Patient statistics}

In this work, the pQA results of 89 wobbling treatments and 44 line-scanning treatments were analyzed. A number of cancer types had been treated and the distribution of disease sites was shown in Table 1 . The patient data was collected from December 2015 to June 2016. For the wobbling method, the major treatment site was the liver, which accounted for half of the total treated fields. For the linescanning method, the main treatment site was CranioSpinal Irradiation (CSI) and its corresponding brain boost, above half (55.1\%) of the total number of fields were included. Pediatric patients had the highest priority for proton therapy because the dosimetric superiority of proton CSI was well-known. ${ }^{14)}$ After that, head and neck cancer treatments made up $26.3 \%$ of the total fields. Only the linescanning method had been used for CSI treatments. For the wobbling method, $48.5 \%$ of the treatment fields were involved liver cancer, and $16.7 \%$ of the fields were for brain cancer.

\section{QA items}

1) QA items for the wobbling method

The pQA for the wobbling method verified the proton range, the width of SOBP. This guaranteed the proton en- ergy selection from the cyclotron, the correct selection of scatterer and ridge filter. The criterion for proton range tolerance was $1 \mathrm{~mm}$. And it was $5 \mathrm{~mm}$ for the width of SOBP. The proton range and the SOBP width were measured by ZEBRA (IBA, Belgium), a multi-layer ion chamber which has $2 \mathrm{~mm}$ spatial resolution.

2) QA items for the line-scanning method

For the line-scanning method, multiple proton energies were used to shape the three-dimensional dose distribution with intensity modulation. The best way to assure the accuracy of dose delivering was to measure a complete three-dimensional dose distribution and to compare it with the plan dose distribution. Currently, however, there is no way to perform it for routine pQA procedures due to the lack of a measurement method and time. As a practical solution, two-dimensional dose distributions at multiple depths were verified as other institutes reported. ${ }^{5-9)}$ For gamma analysis of the two-dimensional dose measurement, the passing rate tolerance was determined to be $95 \%$ with the $3 \% / 3$ mm criteria. ${ }^{15)}$

The two-dimensional dose distributions were measured by Octavius 729 XDR (PTW, Germany), an ion chamber array that has a $1 \mathrm{~cm}$ lateral resolution with a $24 \times 24 \mathrm{~cm}^{2}$ maximum measurable field size. ${ }^{16,17)}$ For measurement, three depths were chosen manually which include a shallow depth (mainly 2 or $3 \mathrm{~cm}$ ), middle depth (near the plan isocenter), and distal depth (2 or $3 \mathrm{~cm}$ deeper than middle depth). For the output measurement, a plane-parallel ionization chamber, PPC05 (IBA, Belgium) and PinPoint chamber (PTW31014, PTW, Freiburg, Germany) were used. The absorbed dose is computed via TRS 398 protocol.

Table 1. Patient statistics of wobbling and line-scanning proton therapy at Samsung Medical Center.

\begin{tabular}{|c|c|c|c|c|c|c|c|c|c|c|c|}
\hline & Brain & Eyeball & $\mathrm{H} \& \mathrm{~N}$ & Thorax & Pelvis & Prostate & CSI & Liver & Abd & Spine & Total \\
\hline \multicolumn{12}{|l|}{ Wobbling } \\
\hline Field & 38 & 8 & 13 & 21 & 10 & 4 & & 110 & 19 & 4 & 227 \\
\hline Patient & 14 & 3 & 6 & 9 & 5 & 2 & & 36 & 12 & 2 & 89 \\
\hline$\%$ of total field & $16.7 \%$ & $3.5 \%$ & $5.7 \%$ & $9.3 \%$ & $4.4 \%$ & $1.8 \%$ & & $48.5 \%$ & $8.4 \%$ & $1.8 \%$ & \\
\hline \multicolumn{12}{|l|}{ Scanning } \\
\hline Field & 25 & 2 & 31 & 12 & 4 & 4 & 40 & & & & 118 \\
\hline Patient & 11 & 1 & 13 & 6 & 2 & 2 & 9 & & & & 44 \\
\hline$\%$ of total field & $21.2 \%$ & $1.7 \%$ & $26.3 \%$ & $10.2 \%$ & $3.4 \%$ & $3.4 \%$ & $33.9 \%$ & & & & \\
\hline
\end{tabular}

H\&N, head and neck; CSI, Cranio-Spinal Irradiation; Abd, abdomen. 
a

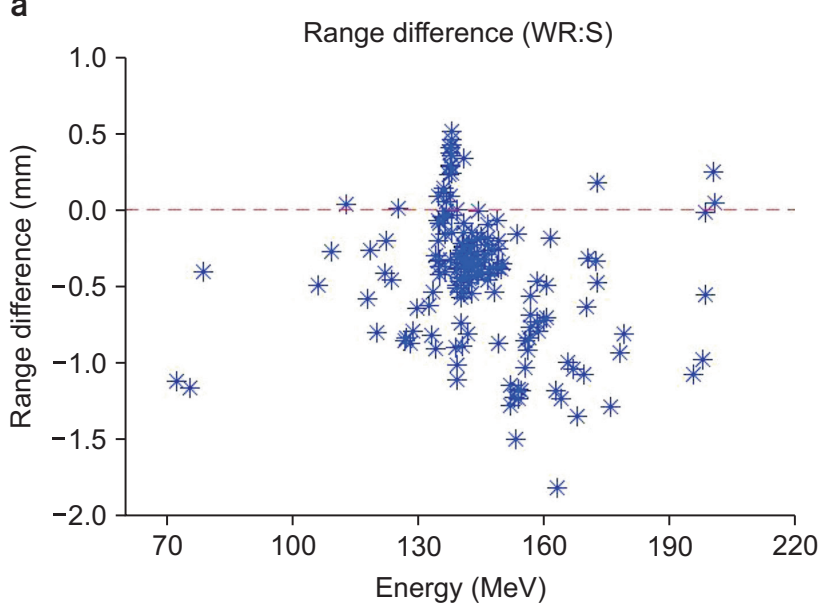

C

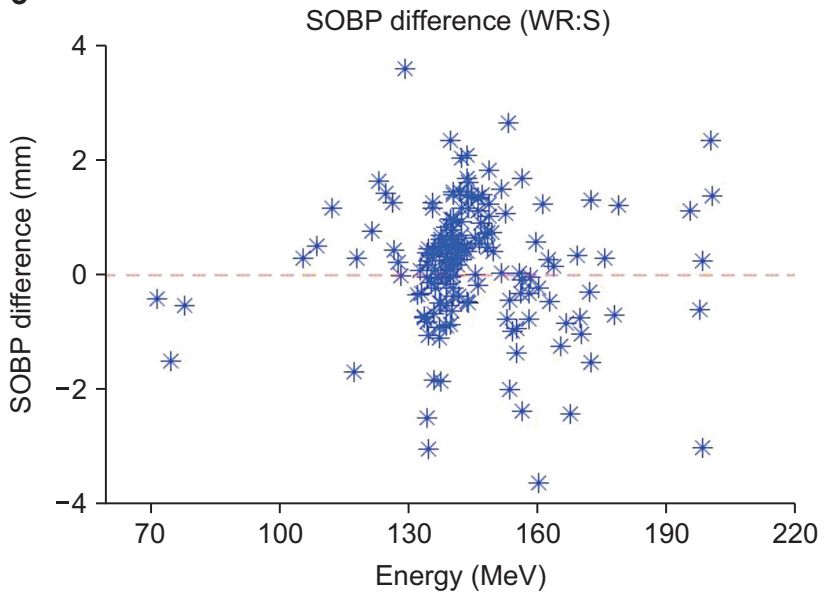

b

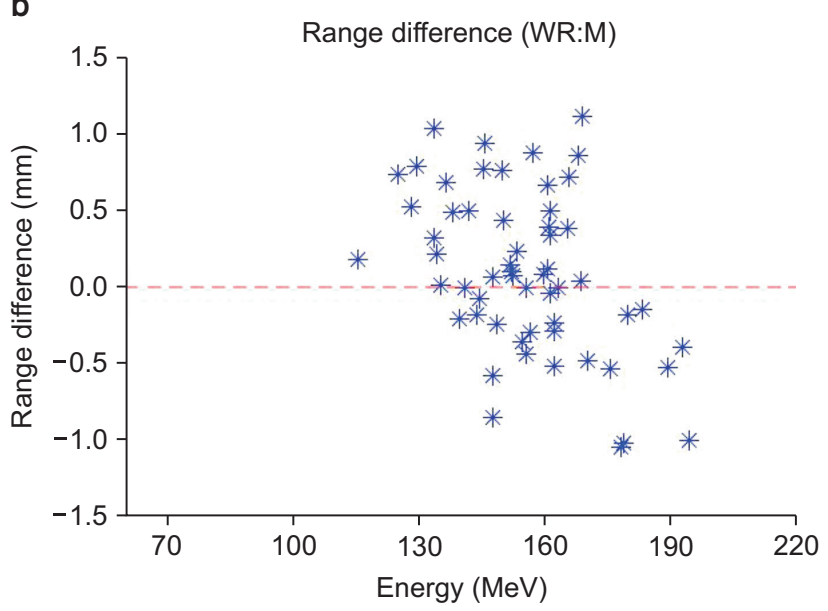

d

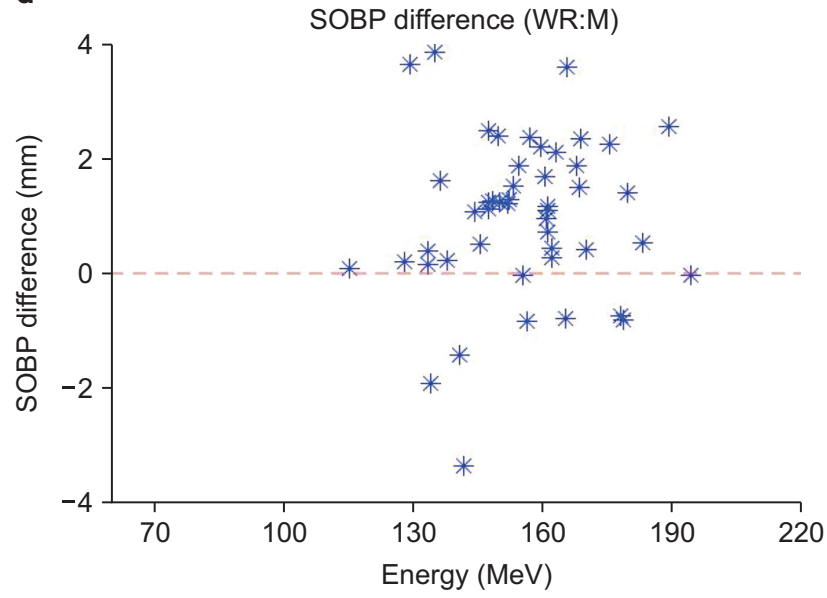

Fig. 2. The difference of range and the SOBP width between the measurement and plan for a small WR and middle WR. (a, b) The difference in range for a small (middle) WR. (c, d) The difference in the SOBP width for a small (middle) WR. WR, wobbling radius; SOBP, spread out Bragg peak.

\section{Results}

\section{Wobbling method}

\section{1) Range and SOBP difference}

The measured range and the SOBP width were categorized into two groups: small WR and middle WR. For a small WR, a total of 168 fields were evaluated. The average difference in proton range was $-0.44 \mathrm{~mm}$ with a standard deviation of $0.43 \mathrm{~mm}$. The average difference in the SOBP width was $0.17 \mathrm{~mm}$ with a standard deviation of $1.64 \mathrm{~mm}$. The frequently used energy was in the interval of $130 \mathrm{MeV}$ to $150 \mathrm{MeV}$, which was for liver cancer as well as head and neck cancer patients. For a middle WR, 59 fields were evaluated. The average difference between a measured and planned proton range was $0.10 \mathrm{~mm}$ with a standard deviation of $0.53 \mathrm{~mm}$. The average difference in the width of SOBP was $1.71 \mathrm{~mm}$ with a standard deviation of $3.91 \mathrm{~mm}$ as seen in Fig. 2.

\section{Line-scanning method}

\section{1) Output difference}

A total 44 plans and 118 treatment fields were analyzed. The output difference presented in Fig. 3 was $-0.69 \%$ on average with a standard deviation of $1.04 \%$. The output difference did not depend on the energy of the first layer or on the measurement depth, which represented the combination of initial energy and the modulation of energy. In order to see the effect of field size on measurement depth, we 
a

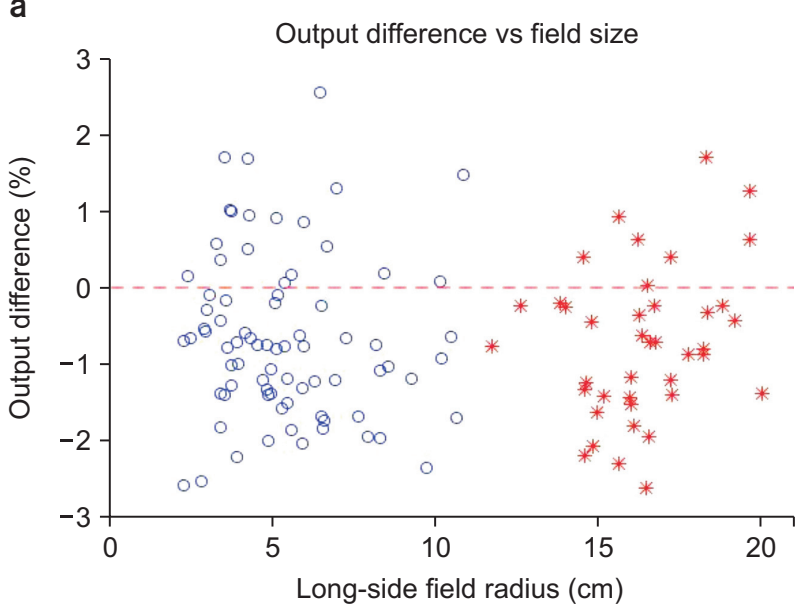

C

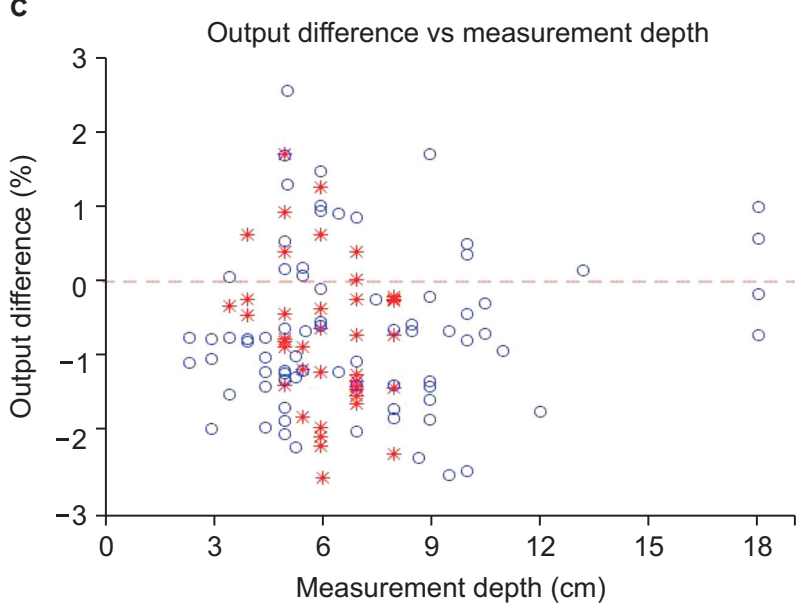

b

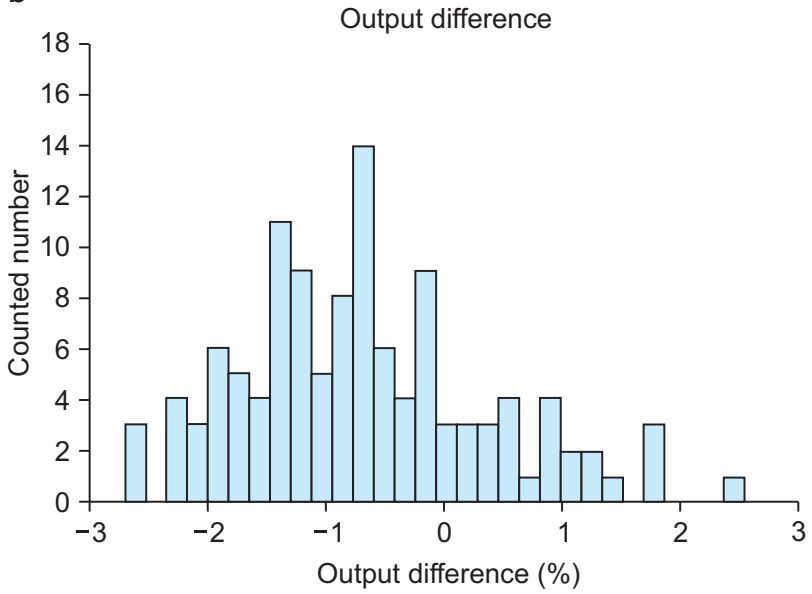

d

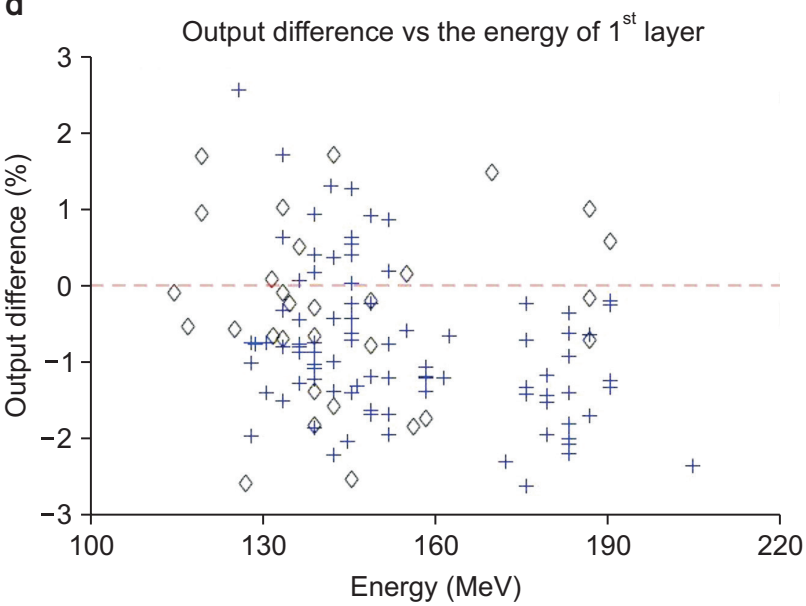

Fig. 3. The output difference between measurement depth and plan as a function of various factors. (a) Long-side field radius vs. output difference, (b) The histogram of output difference, (c) measurement depth vs. output difference, (d) The energy of the first layer with and without range shifter vs. output difference. Blue circle: field size $<11 \mathrm{~cm}$, red asterisk: field size $>11 \mathrm{~cm}$. Blue-plus sign, with range shifter; black diamond, without range shifter.

marked a blue circle for small field sizes (radius less than $11 \mathrm{~cm}$ ) and a red asterisk for large field sizes (radius larger than $11 \mathrm{~cm}$ ) in Fig. 3a, 3c. Both large and small field sizes did not give significant difference. The presence of a range shifter did not have any effect on the output difference, Fig. $3 \mathrm{~d}$ where a plus symbol represented the output with range shifter and a diamond symbol represented those without a range shifter.

\section{2) Two-dimensional dose distribution}

The gamma passing rates for each field were represented as blue circles for the $3 \% / 3 \mathrm{~mm}$ criterion in Fig. 4 . To see the effect of range shifter, a plus symbol represented the output with range shifter and a diamond symbol repre- sented those without a range shifter in Fig. 4. The normalization of output was done at the middle depth, and the normalization factor was applied to the shallow and deep depth measurements to maintain the same three-dimensional dose scaling. At shallow, middle, and deep depths, every field passed the $3 \% / 3 \mathrm{~mm}$ criterion. The passing rates were better at the middle depth than at those at shallow or deep depths.

\section{Discussion}

\section{Wobbling method}

The average range difference was $-0.4 \mathrm{~mm}$ for a small 

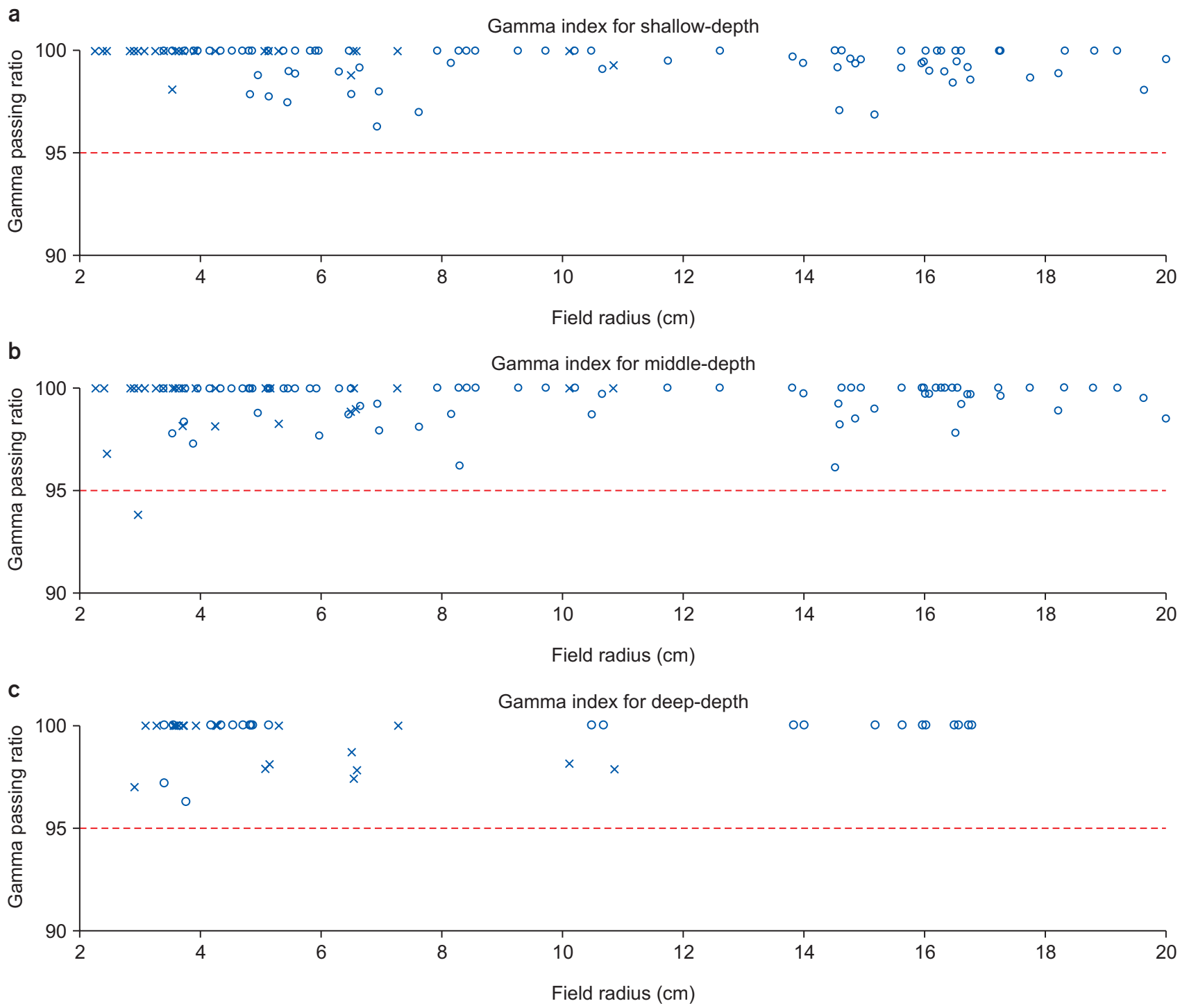

Fig. 4. Gamma index for line-scanning at three depths as a function of field radius. (a) shallow depth, (b) middle depth, (c) deep depth (Blue circle: gamma criterion of $3 \mathrm{~mm} / 3 \%$ ).

WR and $0.1 \mathrm{~mm}$ for a middle WR. If the range difference between a measurement and the treatment plan was less than $1 \mathrm{~mm}$, that was accepted because $1 \mathrm{~mm}$ range uncertainty was already considered in treatment planning margin. There was a small window of proton energy from 150 $\mathrm{MeV}$ to $160 \mathrm{MeV}$. In the window, the range differences of some fields exceeded $1 \mathrm{~mm}$. The discrepancy was caused by beam modelling accuracy and measurement error. After the measurement, we added more range margin in the window because we cannot change any beam parameter like BCM modification of IBA system.

The average difference in the SOBP width was small but the standard deviation was relatively large, with $1.6 \mathrm{~mm}$ for the small WR and $1.7 \mathrm{~mm}$ for the middle WR. This relatively large standard deviation is a characteristic of slowly varying proximal dose distribution of the SOBP. While the proton range was defined consistently due to the steep dose gradient of the distal dose distribution, the proximal 95\% of the SOBP, however, could be 2 or $3 \mathrm{~mm}$ shifted for a small dose variation. In fact, variations of a few millimeters in the SOBP width did not affect the dose coverage of the target. Therefore, the difference in the SOBP width of $5 \mathrm{~mm}$ could be accepted in most cases after reviewing the dose conformity of the target. 


\section{Line-scanning method}

The average output difference was $-0.69 \%$ with a standard deviation of $1.04 \%$. The output difference did not depend on the presence of a range shifter, field size, energy, or modulation width. This small deviation was not only from the excellent beam modelling in TPS but from the daily output correction in the Treatment Control System (TCS). The daily output measurement was compared to a reference value and the deviation was registered as a correction factor in the TCS. The correction factor was applied for each treatment field in order to compensate the output fluctuation of the day. And, thanks to the output correction function in the TCS, the measured output deviation was compensated by the TCS for patient treatment. The actual output difference for each patient should be smaller than this result. At three depths, gamma passing rates with 3\%/3 $\mathrm{mm}$ criterion were over $95 \%$ for every field. From the analysis of 188 scanning fields, we concluded that the beam delivery was within a tolerance and it was greatly matched with the dose computation in homogeneous medium.

\section{Conclusion}

The purpose of patient specific QA is to assess whether the delivered dose would be within the tolerance compared with the patient treatment plan. In addition, another important aspect is to verify that functioning of all integral parts of the treatment system specific to each treatment plan, e.g., a hardware status that includes the selection of the designated scatterer, a ridge filter, together with the scanning magnet performance. In addition, software status e.g. a transfer of treatment planning information via record and verify system, correct scanning pattern downloading and all integrated system software parameter specific to each treatment plan are required to be tested.

The result of pQA for the line-scanning cases was acceptable for patient treatment. For wobbling treatments, the range accuracy and the SOBP width were all acceptable. To enhance the dose computation accuracy, we have a plan to adopt Monte Carlo dose engine in the TPS for wobbling method.

\section{Acknowledgements}

This research is partly supported by a grant from National Research Fund (NRF-2017R1D1A1B04030619 and NRF2013M2A2A7043507).

\section{Conflicts of Interest}

The authors have nothing to disclose.

\section{Availability of Data and Materials}

All relevant data are within the paper and its Supporting Information files.

\section{Ethics Approval and Consent to Participate}

The study was approved by the institutional review board (IRB approval number; 2017-11-017-001).

\section{References}

1. PTCOG-PTCOG Patient Statistics. Ptcogch. 2017. URL: https://www.ptcog.ch/index.php/ptcog-patient-statistics.

2. Chang JY, Zhang X, Wang X, Kang Y, Riley B, Bilton S, Mohan R, Komaki R, Cox JD. Significant reduction of normal tissue dose by proton radiotherapy compared with threedimensional conformal or intensity-modulated radiation therapy in Stage I or Stage III non-small-cell lung cancer. Int J Radiat Oncol Biol Phys. 2006;65:1087-96.

3. Hall EJ. Intensity-modulated radiation therapy, protons, and the risk of second cancers. Int J Radiat Oncol Biol Phys. 2006;65:1-7.

4. Paganetti H. Range uncertainties in proton therapy and the role of Monte Carlo simulations. Phys Med Biol. 2012; 57:R99-117.

5. Lomax AJ, Bohringer T, Bolsi A, Coray D, Emert F, Goitein G, Jermann M, Lin S, Pedroni E, Rutz H, Stadelmann O, Timmermann B, Verwey J, Weber DC. Treatment planning and verification of proton therapy using spot scanning: initial experiences. Med Phys. 2004;31:3150-7.

6. Furukawa T, Inaniwa T, Hara Y, Mizushima K, Shirai T, Noda K. Patient-specific QA and delivery verification of 
scanned ion beam at NIRS-HIMAC. Med Phys. 2013;40: 121707.

7. Mackin D, Zhu XR, Poenisch F, Li H, Sahoo N, Kerr M, Holmes C, Li Y, Lii M, Wu R, Suzuki K, Gillin MT, Frank SJ, Grosshans D, Zhang X. Spot-scanning proton therapy patient-specific quality assurance: results from 309 treatment plans. Int J Part Ther. 2014;1:711-20.

8. Zhu XR, Poenisch F, Song X, Johnson JL, Ciangaru G, Taylor MB, Lii M, Martin C, Arjomandy B, Lee AK, Choi S, Nguyen QN, Gillin MT, Sahoo N. Patient-specific quality assurance for prostate cancer patients receiving spot scanning proton therapy using single-field uniform dose. Int J Radiat Oncol Biol Phys. 2011;81:552-9.

9. Mackin D, Li Y, Taylor MB, Kerr M, Holmes C, Sahoo N, Poenisch F, Li H, Lii J, Amos R, Wu R, Suzuki K, Gillin MT, Zhu XR, Zhang X. Improving spot-scanning proton therapy patient specific quality assurance with HPlusQA, a second-check dose calculation engine. Med Phys. 2013;40: 121708.

10. Chung K, Han Y, Kim J, Ahn SH, Ju SG, Jung SH, Chung Y, Cho S, Jo K, Shin EH, Hong CS, Shin JS, Park S, Kim DH, Kim HY, Lee B, Shibagaki G, Nonaka H, Sasai K, Koyabu Y, Choi C, Huh SJ, Ahn YC, Pyo HR, Lim DH, Park HC, Park W, Oh DR, Noh JM, Yu JI, Song S, Lee JE, Lee B, Choi DH. The first private-hospital based proton therapy center in Korea; status of the Proton Therapy Center at Samsung Medical Center. Radiat Oncol J. 2015;33:337-43.
11. Yonai S, Kanematsu N, Komori M, Kanai T, Takei Y, Takahashi O, Isobe $\mathrm{Y}$, Tashiro M, Koikegami H, Tomita H. Evaluation of beam wobbling methods for heavy-ion radiotherapy. Med Phys. 2008;35:927-38.

12. Torikoshi M, Minohara S, Kanematsu N, Komori M, Kanazawa M, Noda K, Miyahara N, Itoh H, Endo M, Kanai T. Irradiation system for HIMAC. J Radiat Res. 2007;48 Suppl A:A15-25.

13. Haberer T, Becher W, Schardt D, Kraft G. Magnetic scanning system for heavy ion therapy. Nucl Instrum Methods Phys Res A. 1993;330:296-305.

14. St Clair WH, Adams JA, Bues M, Fullerton BC, La Shell S, Kooy HM, Loeffler JS, Tarbell NJ. Advantage of protons compared to conventional X-ray or IMRT in the treatment of a pediatric patient with medulloblastoma. Int J Radiat Oncol Biol Phys. 2004;58:727-34.

15. Low DA, Dempsey JF. Evaluation of the gamma dose distribution comparison method. Med Phys. 2003;30:245564.

16. Arjomandy B, Sahoo N, Ding X, Gillin M. Use of a twodimensional ionization chamber array for proton therapy beam quality assurance. Med Phys. 2008;35:3889-94.

17. Arjomandy B, Sahoo N, Ciangaru G, Zhu R, Song X, Gillin M. Verification of patient-specific dose distributions in proton therapy using a commercial two-dimensional ion chamber array. Med Phys. 2010;37:5831-7. 